hep-th/0006160

CALT-68-2283

CITUSC/00-027

\title{
Quantum Field Theories With Compact Noncommutative Extra Dimensions
}

\author{
Jaume Gomis, Thomas Mehen and Mark B. Wise \\ Department of Physics, California Institute of Technology, Pasadena, CA 91125 \\ and \\ Caltech-USC Center for Theoretical Physics \\ University of Southern California \\ Los Angeles, CA 90089 \\ gomis, mehen, wise@theory.caltech.edu
}

We study field theories on spaces with additional compact noncommutative dimensions. As an example, we study $\phi^{3}$ on $R^{1,3} \times T_{\theta}^{2}$ using perturbation theory. The infrared divergences in the noncompact theory give rise to unusual dynamics for the mode of $\phi$ which is constant along the torus. Correlation functions involving this mode vanish. Moreover, we show that the spectrum of Kaluza-Klein excitations can be very different from the analogous commuting theory. There is an additional contribution to the Kaluza-Klein mass formula that resembles the contribution of winding states in string theory. We also consider the effect of noncommutativity on the four dimensional Kaluza-Klein excitations of a six dimensional gauge field.

June 2000 


\section{Introduction}

Quantum field theories on noncommutative spaces - usually referred to as noncommutative field theories - generalize the familiar structure of conventional (commutative) local quantum field theories. Field theories on these spaces can be studied using conventional techniques by representing the underlying noncommutative structure that defines the noncommutative space in terms of more familiar entities in a commutative space. For

example, noncommutative Minkowski space $R_{\theta}^{1, d}$ is defined in terms of space-time coordinates $x^{\mu}, \mu=0, \ldots, d$, which satisfy the following commutation relations

$$
\left[x^{\mu}, x^{\nu}\right]=i \theta^{\mu \nu} \quad \mu, \nu=0, \ldots, d,
$$

where $\theta^{\mu \nu}$ is a real antisymmetric matrix. $R_{\theta}^{1, d}$ is a noncommutative associative algebra, with elements given by ordinary continuous functions on $R^{1, d}$ and whose product is given by the Moyal bracket or $\star$-product of functions

$$
(f \star g)(x)=\left.e^{\frac{i}{2} \theta^{\mu \nu} \frac{\partial}{\partial \alpha^{\mu}} \frac{\partial}{\partial \beta^{\nu}}} f(x+\alpha) g(x+\beta)\right|_{\alpha=\beta=0}=f g+\frac{i}{2} \theta^{\mu \nu} \partial_{\mu} f \partial_{\nu} g+\mathcal{O}\left(\theta^{2}\right) .
$$

The generalization of conventional field theories on $R^{1, d}$ to $R_{\theta}^{1, d}$ is achieved by replacing the usual multiplication of fields in the action by the $\star$-product of fields.

The presence of $\star$-products in the action leads to theories that are not maximally Lorentz invariant nor local. The nonlocality of the theory is apparent from the infinite number of derivatives that appear in the action. The noncommutativity of the space-time coordinates (1.1) gives rise to a space-time uncertainty relation

$$
\Delta x^{\mu} \Delta x^{\nu} \geq \frac{1}{2}\left|\theta^{\mu \nu}\right| .
$$

A dramatic consequence of this relation is the absence of decoupling of scales in these theories [1]-[5]. For instance, if $\theta^{12} \neq 0$, short distance scales in the $x^{1}$ direction correspond to very large distance scales in the $x^{2}$ direction. Therefore, these theories exhibit a very peculiar mixing between the ultraviolet and the infrared, such that very high energy modes have drastic effects on low energy processes. In spite of this, the theories are apparently renormalizable [6]-[17]. Morever, field theories with only space noncommutativity (that is, $\theta^{0 i}=0$ ) have a unitary S-matrix. On the other hand, theories with only space-time noncommutativity (that is $\theta^{0 i} \neq 0$ ) are not unitary [18].

A strong motivation for understanding these field theories is the appearance they make in string theory [19]-[25]. For instance, noncommutative gauge theories with space 
noncommutativity describe the low energy excitations of open strings on D-branes in a background Neveu-Schwarz two-form field $B$ [19] [20]|21]. The excited open string states and the closed strings decouple and the noncommutative field theory is the proper description of the physics. The lack of covariance of noncommutative field theory arises from the expectation value of $B$. The appearance of noncommutative field theory in a definite limit of string theory strongly suggests that these field theories are sensible quantum field theories. This motivates generalizing the conventional framework of local quantum field theory in order to understand these theories.

In nature we observe three spatial dimensions. However, it is possible that there are additional spatial dimensions. Such is the case in string theory and recently there has been speculation that extra dimensions may play a role in understanding the hierarchy puzzle [26] or the cosmological constant problem [27]. If extra dimensions exist the simplest way to explain why they have not been observed is to assume they are compact. It is possible that such additional compact spatial dimensions are noncommuting. In this paper we explore this possibility in some simple field theories that contain two additional compact noncommuting dimensions. We find that the UV-IR mixing that exists for infinite noncommuting dimensions leads to some interesting properties of the compactified theories.

In this paper we study in perturbation theory scalar and gauge quantum field theories with compact noncommutative extra dimensions. We examine the low energy dynamics of these theories and their spectrum of Kaluza-Klein excitations. For concreteness, we consider six dimensional field theories, such that the two extra dimensions are compact and noncommutative and four noncompact dimensions are commutative. The two extra dimensions are taken to correspond to a noncommutative two torus $T_{\theta}^{2}$ whose coordinates satisfy

$$
\left[x^{4}, x^{5}\right]=i \theta
$$

This system can be realized in string theory by wrapping a five-brane on a two-torus $T^{2}$ with a constant $B$-field along the torus. The low energy effective four dimensional theory resulting from compactification on a noncommutative space is local and Lorentz invariant, hence it can be relevant phenomenologically.

In section 2 we consider scalar $\phi^{3}$ theory on $R^{1,3} \times T_{\theta}^{2}$. The noncommutative theory on a noncompact space contains infrared divergences. In the compact theory these appear as additional divergences in two and three point functions involving the mode of $\phi$ that is constant along the torus, $\phi_{0}$. The correct interpretation of these divergences is unusual 
dynamics for $\phi_{0}$. Since the mass of $\phi_{0}$ diverges as the ultraviolet cutoff is taken to infinity, $\phi_{0}$ no longer appears as a propagating degree of freedom in the low energy effective theory.

In section 3 we discuss the Kaluza-Klein mass formula for the scalar field theory introduced in section 2. There are one loop corrections which are singular as $\theta$ goes to zero. For small $\theta$, they lead to a four dimensional spectrum of states which differs qualitatively from the Kaluza-Klein spectrum of a commutative theory. The one loop correction to the dispersion relation for the Kaluza-Klein excitations resembles that of winding states in string theory [四].

In section 4 we briefly discuss similar issues in a $U(1)$ gauge theory compactified on $R^{1,3} \times T_{\theta}^{2}$. Again we find that modes of the gauge field which are constant along the torus disappear from the low energy theory. The six dimensional gauge field contains KaluzaKlein excitations that are either four-dimensional vectors or four-dimensional scalars. One loop corrections modify the spectrum of the four-dimensional scalars, however, the spectrum of the four-dimensional vectors is unchanged.

Concluding remarks are given in section 5 .

\section{Perturbation Theory for $\phi^{3}$ on $R^{1,3} \times T_{\theta}^{2}$}

The noncommutative scalar $\phi^{3}$ theory in six noncompact dimensions is defined by the following action:

$$
S=\int d^{6} x\left(\frac{1}{2}(\partial \phi)^{2}-\frac{1}{2} m^{2} \phi^{2}-\frac{\lambda}{3 !} \phi \star \phi \star \phi\right) .
$$

The coordinates of commutative four dimensional Minkowski space are $x^{0}, x^{1}, x^{2}, x^{3}$. The coordinates $x^{4}$ and $x^{5}$ are noncommuting :

$$
\left[x^{4}, x^{5}\right]=i \theta \text {. }
$$

Since the theory defined by (2.1) contains an infinite number of higher derivative operators, naively one would expect it to be nonrenormalizable. However, it is a renormalizable theory because all ultraviolet divergences can be cancelled by counterterms of the form appearing in the action (2.1). Unlike conventional field theories, the counterterms are not local operators but the nonlocality is of the same type present in the tree level action. The S-matrix elements of this theory are plagued with infrared divergences. For instance, the 
one loop correction to the two point function leads to a term in the effective action of the form

$$
-\int d^{6} x \frac{\lambda^{2}}{2(4 \pi)^{3}} \phi \frac{1}{\partial \circ \partial} \phi
$$

where we have introduced the inner product $p \circ p=-p_{M}\left(\theta^{2}\right)^{M N} p_{N}=\theta^{2}\left(p_{4}^{2}+p_{5}^{2}\right)$.

The action in (2.1) must be augmented by cutoff dependent counterterms. In this paper we work at lowest nontrivial order in perturbation theory (i.e. order $\lambda^{2}$ in 1PI diagrams) and ignore the obvious classical instability in (2.1). The one loop counterterms in the noncompact theory are

$$
S_{c t}=-\int d^{6} x\left(\frac{\lambda^{2}}{8(4 \pi)^{3}}\left(\Lambda^{2}-m^{2} \ln \Lambda^{2}\right) \phi^{2}+\frac{\lambda^{2}}{48(4 \pi)^{3}} \ln \Lambda^{2}(\partial \phi)^{2}+\frac{\lambda^{3}}{48(4 \pi)^{3}} \ln \Lambda^{2} \phi \star \phi \star \phi\right) .
$$

The explicit form of the ultraviolet regulator $\Lambda$ will be discussed later.

In this paper, we will be concerned with the theory on a space with two noncommuting compact directions. The divergence structure of the noncommutative theory on a compact space is different from that of the theory on a noncompact space. The coordinates $x^{4}$ and $x^{5}$ are compactified on a rectangular torus with $0 \leq x^{4}, x^{5} \leq 2 \pi R$. Momenta along the compact directions are quantized, $\vec{p}=\vec{n} / R$, where $\vec{n}=\left(n_{4}, n_{5}\right)$ are integers. For the mode with $\vec{n} \neq 0$, the term in the one particle irreducible action shown in (2.3) is finite. It gives rise to an interesting modification of the Kaluza-Klein spectrum that will be discussed in section 3. For the mode with $\vec{n}=0$, (2.3) is infinite.

Given the special role played by $\vec{n}=0$ mode, it is very useful to make the following definitions

$$
\begin{aligned}
\phi_{0} & =\frac{1}{(2 \pi R)^{2}} \int d x^{4} d x^{5} \phi \\
\bar{\phi} & =\phi-\phi_{0} .
\end{aligned}
$$

The $\phi_{0}$ field contains the mode with $\vec{n}=0, \bar{\phi}$ contains all modes with nonvanishing $\vec{n}$. In terms of these fields the action (2.1) is:

$$
S=\int d^{6} x\left(\frac{1}{2}(\partial \bar{\phi})^{2}-\frac{1}{2} m^{2} \bar{\phi}^{2}+\frac{1}{2}\left(\partial \phi_{0}\right)^{2}-\frac{1}{2} m^{2} \phi_{0}^{2}-\frac{\lambda}{3 !} \bar{\phi} \star \bar{\phi} \star \bar{\phi}-\frac{\lambda}{2} \bar{\phi}^{2} \phi_{0}-\frac{\lambda}{3 !} \phi_{0}^{3}\right) .
$$

The products involving $\phi_{0}$ are ordinary products. This is because after integration the $\star$-product of three fields reduces to an ordinary product whenever one of the fields has vanishing momentum along noncommuting dimensions. No operator of the form $\int d^{6} x \bar{\phi} \phi_{0}^{2}$ appears because it is forbidden by momentum conservation. 

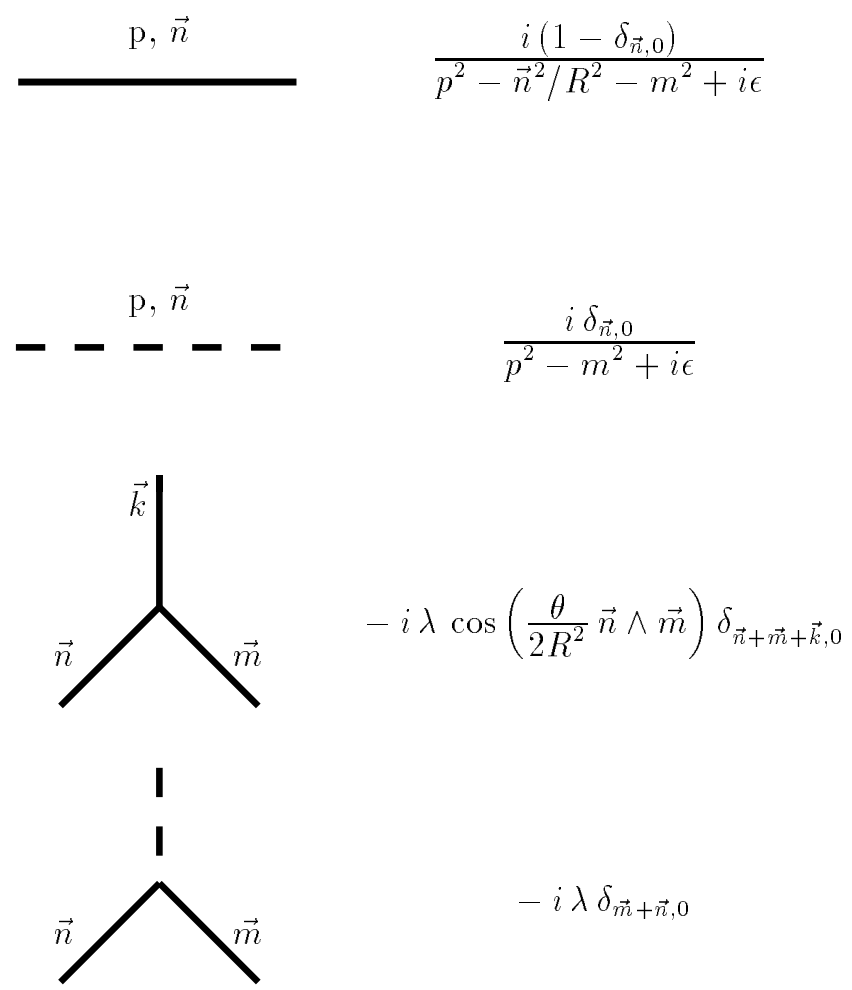

$$
-i \lambda \delta_{\vec{m}+\vec{n}, 0}
$$

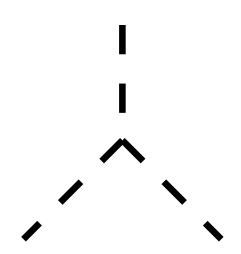

$-i \lambda$

Fig. 1: Feynman rules for noncommutative $\phi^{3}$ on $R^{1,3} \times T_{\theta}^{2}$. Solid lines are $\bar{\phi}$ quanta, dotted lines are $\phi_{0}$ quanta. Momenta along noncompact directions are denoted by $p$, while $\vec{n} / R, \vec{k} / R$ and $\vec{m} / R$ are momenta along compact directions. The wedge product is defined to be $\vec{n} \wedge \vec{k} \equiv n_{4} k_{5}-n_{5} k_{4}$.

The Feynman rules derived from the action (2.6) are given in fig. 1. Noncommutativity introduces oscillatory factors in the vertices of the theory. These oscillatory factors never make a graph more ultraviolet divergent than the naive power counting estimate. Therefore if a graph is ultraviolet finite in the commutative theory it will also be ultraviolet finite in the noncommutative theory. Since $\phi^{3}$ in six dimensions is renormalizable, only the two 
and three point functions of this theory can have ultraviolet divergences.

a)

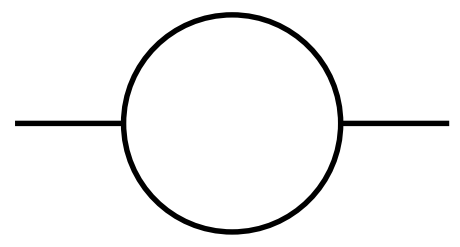

c)

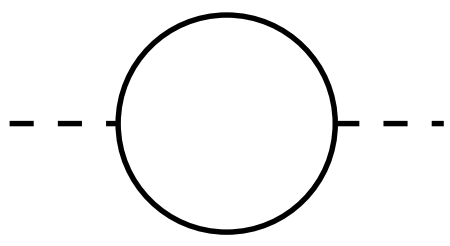

b)

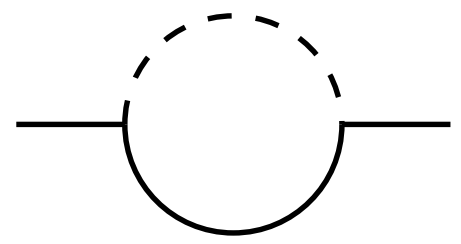

d)

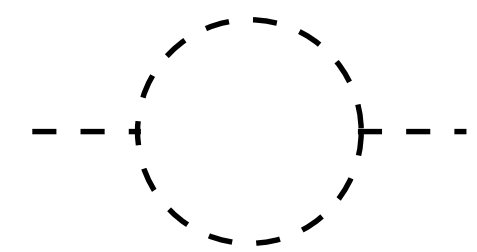

Fig. 2: One loop corrections to the two point functions for $\phi_{0}$ and $\bar{\phi}$.

The one loop contributions to the two point functions are shown in fig. 2. We begin with diagram $a$ ), which is a contribution to the two point function of $\bar{\phi}$.

$$
a)=\frac{\lambda^{2}}{2} \frac{1}{(2 \pi R)^{2}} \sum_{\vec{k}} \int \frac{d^{4} l}{(2 \pi)^{4}} \frac{\cos ^{2}\left(\theta \vec{n} \wedge \vec{k} /\left(2 R^{2}\right)\right)\left(1-\delta_{\vec{k}, 0}\right)\left(1-\delta_{\vec{n}+\vec{k}, 0}\right)}{\left(l^{2}-\vec{k}^{2} / R^{2}-m^{2}\right)\left((l+p)^{2}-(\vec{n}+\vec{k})^{2} / R^{2}-m^{2}\right)}
$$

In (2.7), $l$ denotes the loop momenta along the noncompact directions, while $\vec{k} / R$ is loop momenta along compact directions. Similarly, $p(\vec{n} / R)$ is the external momenta along the noncompact (compact) directions. In (2.7), $\vec{n} \wedge \vec{k} \equiv n_{4} k_{5}-n_{5} k_{4}$. Since the external field is $\bar{\phi}, \vec{n} \neq 0$. This implies $\delta_{\vec{k}, 0} \delta_{\vec{n}+\vec{k}, 0}=0$. We can simplify the numerator by using the half angle formula for the cosine and the fact that $\vec{n} \wedge \vec{k}=0$ for $\vec{k}=0,-\vec{n}$ to obtain:

$$
a)=\frac{\lambda^{2}}{4} \frac{1}{(2 \pi R)^{2}} \sum_{\vec{k}} \int \frac{d^{4} l}{(2 \pi)^{4}} \frac{1-2 \delta_{\vec{k}, 0}-2 \delta_{\vec{n}+\vec{k}, 0}+\cos \left(\theta \vec{n} \wedge \vec{k} / R^{2}\right)}{\left(l^{2}-\vec{k}^{2} / R^{2}-m^{2}\right)\left((l+p)^{2}-(\vec{n}+\vec{k})^{2} / R^{2}-m^{2}\right) .}
$$

The oscillatory factor $\cos \left(\theta \vec{n} \wedge \vec{k} / R^{2}\right)$ makes the last term of (2.8) ultraviolet finite. The first term is quadratically divergent, while the second and third terms are logarithmically divergent. Only the divergent terms will be evaluated in this section. 
We first represent the propagator using Schwinger parameters then perform the Gaussian integral over the loop momentum to obtain

$$
\begin{array}{r}
a)=\frac{i \lambda^{2}}{4(4 \pi)^{3} \pi R^{2}} \int_{0}^{\infty} \frac{d \alpha}{\alpha} \int_{0}^{1} d x\left(\sum_{\vec{k}} \exp \left[-\alpha\left(m^{2}-x(1-x) p^{2}+\frac{\vec{k}^{2}}{R^{2}}+x \frac{\vec{n}^{2}+2 \vec{n} \cdot \vec{k}}{R^{2}}\right)\right]\right. \\
\left.-4 \exp \left[-\alpha\left(m^{2}-x(1-x) p^{2}+x \frac{\vec{n}^{2}}{R^{2}}\right)\right]\right) .
\end{array}
$$

The sum over $\vec{k}$ is performed using the definition of the Jacobi theta function

$$
\vartheta(\nu, \tau)=\sum_{n=-\infty}^{\infty} \exp \left(\pi i n^{2} \tau+2 i \pi n \nu\right)
$$

and the modular transformation $\vartheta(\nu, \tau)=(-i \tau)^{-1 / 2} \exp \left(-\pi i \nu^{2} / \tau\right) \vartheta(\nu / \tau,-1 / \tau)$. The result is

$$
\begin{array}{r}
\frac{i \lambda^{2}}{4(4 \pi)^{3}}\left\{\int_{0}^{\infty} \frac{d \alpha}{\alpha^{2}} \int_{0}^{1} d x \exp \left[-\alpha\left(m^{2}+x(1-x)\left(-p^{2}+\frac{\vec{n}^{2}}{R^{2}}\right)\right)\right] \hat{\vartheta}\left(x n_{4}\right) \hat{\vartheta}\left(x n_{5}\right)\right. \\
\left.-\frac{4}{\pi R^{2}} \int_{0}^{\infty} \frac{d \alpha}{\alpha} \int_{0}^{1} d x \exp \left[-\alpha\left(m^{2}-x(1-x) p^{2}+x \frac{\vec{n}^{2}}{R^{2}}\right)\right]\right\} .
\end{array}
$$

In this formula we have defined $\hat{\vartheta}(\nu)=\vartheta\left(\nu, i \pi R^{2} / \alpha\right)$. The ultraviolet divergent contribution comes from the $\alpha \rightarrow 0$ region and in this limit we can set the $\hat{\vartheta}$ functions to unity. Then the first term is proportional to the quadratically divergent one loop correction in the commutative theory in the $R \rightarrow \infty$ limit. Corrections to this limit come from the $n \neq 0$ terms in the expansion of $(2.10)$ and these are finite. There are also corrections arising because of the absence of the $\vec{n}=0$ mode in the loop integral. These corrections depend explicitly on the size of the compact dimension and are logarithmically divergent since the loop integral is effectively four dimensional for the zero mode.

The integrals can be regulated by inserting into the integral $\exp \left(-1 /\left(\Lambda^{2} \alpha\right)\right)$, where $\Lambda$ is the ultraviolet cutoff. The divergent contribution from diagram $a$ ) in fig. 2 is:

$$
a)=\frac{i \lambda^{2}}{4(4 \pi)^{3}}\left[\Lambda^{2}-\left(m^{2}+\frac{1}{6}\left(-p^{2}+\frac{\vec{n}^{2}}{R^{2}}\right)+\frac{4}{\pi R^{2}}\right) \ln \Lambda^{2}\right] .
$$

The divergences in the other graphs in fig. 2 can be computed in the same way. We find

$$
\begin{aligned}
& \text { b) }=\frac{i \lambda^{2}}{(4 \pi)^{3}} \frac{\ln \Lambda^{2}}{\pi R^{2}} \\
& \text { c) }=\frac{i \lambda^{2}}{2(4 \pi)^{3}}\left[\Lambda^{2}-\left(m^{2}-\frac{p^{2}}{6}+\frac{1}{\pi R^{2}}\right) \ln \Lambda^{2}\right] \\
& \text { d) }=\frac{i \lambda^{2}}{2(4 \pi)^{3}} \frac{\ln \Lambda^{2}}{\pi R^{2}} .
\end{aligned}
$$


a)

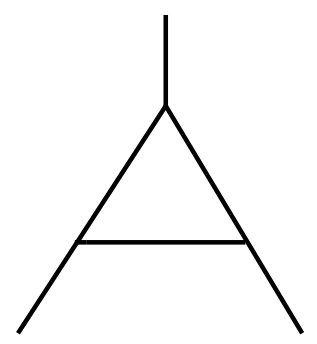

b)

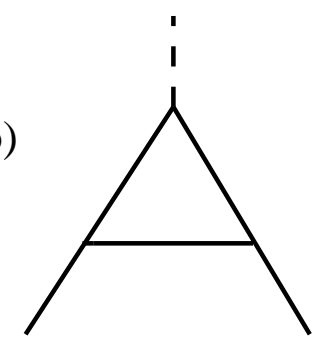

c)

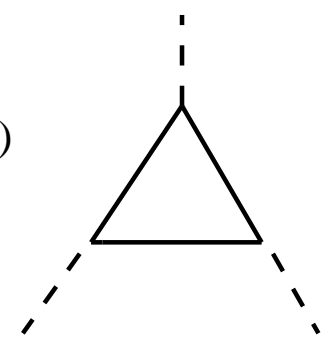

Fig. 3: Divergent one loop corrections to the three point functions.

Next we consider the one loop divergences in the three point functions of the theory. If a zero mode appears in the loop then the loop integration is effectively four dimensional and the graph is finite by power counting. Therefore, it is only necessary to consider loop graphs with internal $\bar{\phi}$ quanta. The relevant Feynman graphs are shown in fig. 3. The leading divergences are logarithmic and these can be obtained from a calculation treating the loop momentum as continuous. The divergences in the graphs of fig. 3 are

$$
\begin{aligned}
& a)=-\frac{i \lambda^{3}}{8(4 \pi)^{3}} \cos \left(\frac{\theta}{2 R^{2}} \vec{n} \wedge \vec{k}\right) \ln \Lambda^{2}, \\
& b)=-\frac{i \lambda^{3}}{4(4 \pi)^{3}} \ln \Lambda^{2} \\
& c)=-\frac{i \lambda^{3}}{2(4 \pi)^{3}} \ln \Lambda^{2} .
\end{aligned}
$$

The two and three point functions for $\bar{\phi}$ are rendered finite by adding the counterterms in (2.4). These counterterms do not render correlation functions involving $\phi_{0}$ finite in the compactified theory. After adding the counterterms in (2.4), the divergent part of the $\phi_{0}$ self energy is

$$
\Sigma_{0}=-\frac{\lambda^{2}}{4(4 \pi)^{3}}\left[\Lambda^{2}-\left(m^{2}-\frac{p^{2}}{6}\right) \ln \Lambda^{2}\right] .
$$

A similar uncancelled divergence appears in three point functions involving $\phi_{0}$. The counterterm in (2.4) regulates three point involving three $\bar{\phi}$ but not the divergences from diagrams $b$ ) and $c$ ) in fig. 3 .

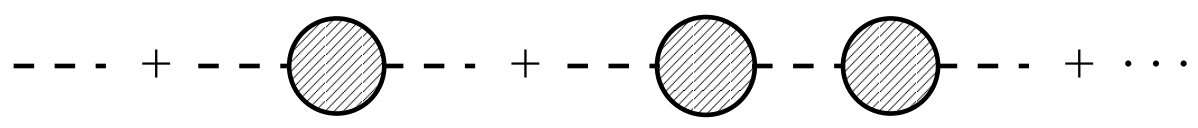

Fig. 4: Resummation of divergent terms in $\phi_{0}$ two-point function. The shaded blob represents $\Sigma_{0}$ in (2.15). 
In the noncompact theory the two and three point functions contain infrared divergences. In the compactified theory, these infrared divergences appear as uncancelled cutoff dependence in two and three point functions involving $\phi_{0}$. The correct interpretation of the uncancelled dependence on $\Lambda$ is obtained by considering renormalization from the Wilsonian point of view. Instead of trying to introduce new counterterms, we interpret the ultraviolet cutoff as a physically significant scale. Then it is clear that the $\phi_{0}$ mode, unlike the $\bar{\phi}$ modes, develops a mass of order $\Lambda$ and hence decouples from the low energy theory as $\Lambda \rightarrow \infty$. This decoupling can be seen diagramatically. As $\Lambda \rightarrow \infty, \Sigma_{0} \rightarrow \infty$. When $\Sigma_{0}$ becomes large it is necessary sum insertions of $\Sigma_{0}$ in the $\phi_{0}$ propagator to all orders, as shown in fig. 4. The two-point correlation function $\left\langle 0\left|T\left(\phi_{0}(x) \phi_{0}(y)\right)\right| 0\right\rangle$ is of order $1 / \Lambda^{2}$ and hence vanishes as $\Lambda^{2} \rightarrow \infty$. Other correlation functions involving $\phi_{0}$ also vanish.

In any correlation function, the resummation shown in fig. 4 is to be performed whenever $\phi_{0}$ is an external line or for graphs which fall apart when a $\phi_{0}$ propagator is cut. Furthermore, at the order in perturbation theory we are working this resummation is only performed for $\phi_{0}$ propagators which do not appear inside 1PI subgraphs. For example, this resummation is not performed for internal $\phi_{0}$ in diagrams $b$ ) and $d$ ) of fig. 2 . This procedure gives finite answers for all correlation functions at this order in perturbation theory. There are uncancelled divergences proportional to $\ln \Lambda^{2}$ in the 1PI three point functions with one or three external $\phi_{0}$. However, the resummed propagators for the $\phi_{0}$ fall off as $1 / \Lambda^{2}$.

Because of the sign of the correction in (2.15), the mass of the zero mode appears to be driven to negative, perhaps indicating that $\phi_{0}$ is tachyonic. Unfortunately, it is impossible to address this issue in the one loop approximation we are employing in this paper. This is because higher order corrections to the self energy are of the form

$$
\delta \Sigma_{0} \sim \lambda^{2 n+2} \Lambda^{2} \ln ^{n} \Lambda
$$

Perturbation theory breaks down for $\Lambda \rightarrow \infty$ and the sign of the quadratic divergence cannot be determined by lowest order perturbation theory. The zero mode mass is likely to be driven to $\pm \infty$, but the sign is not known from the one loop calculation we have performed in this section. At the next order in perturbation theory $\Sigma_{0}$ will occur in loops that renormalize the masses of the $\bar{\phi}$ modes. The cutoff dependence of these corrections must be cancelled by the counterterms if the low energy effective theory has dynamics for the modes of $\bar{\phi}$ that is close to what is expected based on the one loop analysis. 
This completes the analysis of the cutoff dependence of the compactified theory at the one loop level. All necessary counterterms are present in (2.4) and the additional divergences remove $\phi_{0}$ from the low energy theory. An important outstanding issue is to see how ultraviolet divergences cancel at higher orders.

\section{Kaluza-Klein Spectra on Noncommutative Tori}

In this section we compute the spectrum of Kaluza-Klein modes that arises from (2.1) compactified on $T_{\theta}^{2}$. In the classical limit, the $\phi$ quanta of the commutative and noncommutative theories have the same dispersion relation. However, the nonlocality of noncommutative field theories leads to one loop corrections to the dispersion relation. In the noncompact case the one loop dispersion relation is of the form

$$
p^{2}-m^{2}+\frac{\lambda^{2}}{(4 \pi)^{3}} \frac{1}{p \circ p}+\ldots=0,
$$

where the ellipsis denote terms that are less singular as $p \circ p \rightarrow 0$. The nonanalytic dependence in $\theta$ of (3.1) results in corrections that diverge for soft momenta along the noncommutative directions. After compactification, the discretization of momenta isolates the zero momentum mode of the field, such that only for this component of the field one obtains a divergence in the one loop dispersion relation (3.1). As shown in section 2 this quantum correction drives the $\phi_{0}$ mass to infinity, effectively removing $\phi_{0}$ from the theory.

It is straightforward to compute the one loop dispersion relation for the non-zero modes. The relevant Feynman graphs that need to be evaluated are diagrams $a$ ) and $b$ ) of fig. 2. As shown in section 2 , the divergences in these graphs are absorbed by counterterms. We are interested in the finite corrections coming from the nonplanar piece of diagram $a$ ), i.e., the term proportional to $\cos \left(\theta \vec{n} \wedge \vec{k} / R^{2}\right)$ in (2.8). This piece gives the leading behavior for small $\theta$. The one loop self energy is

$$
\begin{aligned}
\Sigma= & -\frac{\lambda^{2}}{4(4 \pi)^{3}} \int_{0}^{1} d x \int_{0}^{\infty} d \alpha \alpha^{-2} \exp \left[-\alpha\left(m^{2}+x(1-x)\left(-p^{2}+\frac{\vec{n}^{2}}{R^{2}}\right)\right)-\frac{\theta^{2} \vec{n}^{2}}{4 R^{2} \alpha}\right] \\
& \times\left\{\frac{1}{2} \hat{\vartheta}\left(x n_{4}+i \frac{\theta n_{5}}{2 \alpha}\right) \hat{\vartheta}\left(x n_{5}-i \frac{\theta n_{4}}{2 \alpha}\right)+\frac{1}{2} \hat{\vartheta}\left(x n_{4}-i \frac{\theta n_{5}}{2 \alpha}\right) \hat{\vartheta}\left(x n_{5}+i \frac{\theta n_{4}}{2 \alpha}\right)\right\} .
\end{aligned}
$$

Setting $p^{2}-\vec{n}^{2} / R^{2}=m^{2}$ in the above formula, the leading terms for small $\theta$ are

$$
\Sigma=-\frac{\lambda^{2}}{(4 \pi)^{3}}\left(\frac{R^{2}}{\theta^{2} \vec{n}^{2}}+\frac{5}{24} m^{2} \ln \left(\frac{m^{2} \theta^{2} \vec{n}^{2}}{R^{2}}\right)+\ldots\right),
$$


so that the formula for the Kaluza-Klein masses is

$$
m_{\vec{n}}^{2}=m^{2}+\frac{\vec{n}^{2}}{R^{2}}-\frac{\lambda^{2}}{(4 \pi)^{3}}\left(\frac{R^{2}}{\vec{n}^{2} \theta^{2}}+\frac{5}{24} m^{2} \ln \left(\frac{m^{2} \theta^{2} \vec{n}^{2}}{R^{2}}\right)+\ldots\right),
$$

Spatial noncommutativity in the compact directions gives a correction to the Kaluza-Klein mass formula which resembles that of winding states in string theory. Note that the mass correction from the nonplanar diagram is negative. For sufficiently small $\theta$, this correction induces a negative mass squared for some Kaluza-Klein modes. The ellipses in Eqs. (3.3) and (3.4) denote terms that are less important as $\theta \rightarrow 0$.

\section{Noncommutative $U(1)$ Gauge Theory in Six Dimensions}

In a noncommutative space-time a pure $U(1)$ gauge theory is interacting. The action in six dimensions is

$$
S=-\frac{1}{4} \int d^{6} x F_{M N} \star F^{M N},
$$

where the field strength tensor

$$
F_{M N}=\partial_{M} A_{N}-\partial_{N} A_{M}-i g\left(A_{M} \star A_{N}-A_{N} \star A_{M}\right),
$$

transforms as

$$
\delta_{\alpha} F_{M N}=i g\left(\alpha \star F_{M N}-F_{M N} \star \alpha\right)
$$

under the $U(1)$ gauge transformation

$$
\delta_{\alpha} A_{M}=\partial_{M} \alpha+i g\left(\alpha \star A_{M}-A_{M} \star \alpha\right) .
$$

Terms in the action quadratic in $A$ are the same as in the commutative theory so the tree level spectrum of Kaluza-Klein excitations follows that case. These are massive four vectors, labelled by $A_{\mu}^{(\vec{n})}$, and scalars, labeled by $h^{(\vec{n})}=\left(n_{5} A_{4}^{(\vec{n})}-n_{4} A_{5}^{(\vec{n})}\right) / \sqrt{\vec{n}^{2}}$, with masses

$$
m_{\vec{n}}^{2}=\frac{\vec{n}^{2}}{R^{2}}
$$

Note that the orthogonal scalar component, $g^{(\vec{n})}=\left(n_{4} A_{4}^{(\vec{n})}+n_{5} A_{5}^{(\vec{n})}\right) / \sqrt{\vec{n}^{2}}$, can be gauged away. It has become the longitudinal component of the massive four vectors $A_{\mu}^{(\vec{n})}$ through the standard Higgs mechanism.

As in the case of scalars discussed in Section 2 the $\vec{n}=0$ modes need to be treated differently. They consist of a four dimensional gauge field and two massless scalars. In this 
case the zero mode fields do not interact with each other nor do they couple to the $\vec{n} \neq 0$ modes. There are no one loop graphs contributing to the two point functions of these fields in the compactified theory. However, these fields receive a divergent wavefunction renormalization from the counterterms of the noncompact theory. This divergence can be removed simply by making a field redefinition. If currents charged under the $\mathrm{U}(1)$ gauge symmetry were added to the theory, the field redefinition would result in a suppression of the couplings of the gauge field to these currents. The $\vec{n}=0$ modes decouple from the low energy effective field theory, not because their masses are driven to infinity (as in the case of the scalar theory), but because their couplings to other fields are driven to zero.

The perturbative corrections to the masses of the Kaluza-Klein modes with $\vec{n} \neq 0$ follow from the two point function $\Pi_{M N}$ of the gauge field. As in the case of the scalar field theory considered in the last section, the most important corrections for $\theta<R^{2}$ can be obtained by replacing the discrete sum over compact loop momenta with a continuum integral. This yields [5] [3] 28]

$$
\Pi_{M N}=\Pi_{M N}^{p}+\Pi_{M N}^{n p}
$$

where 1

$$
\Pi_{M N}^{p}=\frac{i g^{2}}{(4 \pi)^{3}} \int_{0}^{\infty} \frac{d \alpha}{\alpha^{2}} \int_{0}^{1} d x \exp \left[p^{2} \alpha x(1-x)\right]\left[\left(p^{2} g_{M N}-p_{M} p_{N}\right)\left(8-4(1-2 x)^{2}\right)\right]
$$

and

$$
\begin{aligned}
\Pi_{M N}^{n p}=-\frac{i g^{2}}{(4 \pi)^{3}} \int_{0}^{\infty} \frac{d \alpha}{\alpha^{2}} \int_{0}^{1} d x & \exp \left(p^{2} \alpha x(1-x)-\frac{p \circ p}{4 \alpha}\right) \\
\times & {\left[\left(p^{2} g_{M N}-p_{M} p_{N}\right)\left(8-4(1-2 x)^{2}\right)-\frac{4}{\alpha^{2}} \tilde{p}_{M} \tilde{p}_{N}\right] . }
\end{aligned}
$$

Here $\tilde{p}_{M}=\theta_{M N} p^{N}$ and $p$ is the six dimensional external momentum. The components of external momentum along the compact directions, $p_{4}$ and $p_{5}$, are quantized.

The first term inside the brackets in (4.8) gives rise to wavefunction renormalization for the massive vector modes $A_{\mu}^{(\vec{n})}$ and does not change their masses. However, the term

$1 \Pi_{M N}$ is calculated in background field gauge. This amounts to replacing $A_{M}$ in Eq. action by $Q_{M}+A_{M}$ and adding the gauge fixing term $1 / 2 \int d^{6} x G^{2}$, where $G=\partial_{M} Q^{M}-i g\left(A_{M} \star Q^{M}-\right.$ $\left.Q_{M} \star A^{M}\right)$. Note that we are using the convention $g_{\mu \nu}=\operatorname{diag}(+,-,-,-,-,-)$. 
proportional to $\tilde{p}_{M} \tilde{p}_{N}$ can affect the masses of the scalars $h^{(\vec{n})}$. Using the same approximations that were used in the scalar case we find that the Kaluza-Klein spectrum for the $h^{(\vec{n})}$ at one loop is

$$
m_{\vec{n}}^{2}=\frac{\vec{n}^{2}}{R^{2}}-\frac{8 g^{2} R^{4}}{\pi^{3} \theta^{4}\left(\vec{n}^{2}\right)^{2}}+\ldots
$$

Again for small $\theta$ the second term in (4.9) can dominate over the first and drives $m_{\vec{n}}^{2}$ negative. Higher order terms in the effective potential for $h^{(\vec{n})}$ must be computed to determine whether this results in a vacuum expectation value for the $h^{(\vec{n})}$ or signals a true instability of the theory.

\section{Conclusion}

It is a widely held belief that there are additional compactified spatial dimensions. Extra dimensions occur in string theory and it has even been conjectured that their presence may lead to a resolution of the hierarchy puzzle [26] or cosmological constant [27] problem. If extra spatial dimensions do exist then it is possible that they are noncommuting. Motivated by this possibility we have explored the properties of some simple theories with noncommuting compact extra dimensions.

We considered, at lowest order perturbation theory, a $\phi^{3}$ theory compactified on a rectangular noncommuting two torus, $T_{\theta}^{2}$. The UV-IR mixing that occurs in the uncompactified case has important implications for the compactified theory. It causes the mode of the $\phi$ field that is constant on the torus (i.e. the zero mode) to have different dynamics than the other modes. Quantum corrections drive correlation functions of this mode to zero. It does not appear as a propagating degree of freedom in the effective four dimensional theory.

The mass spectrum of Kaluza-Klein excitations was calculated in this theory. At the one loop level the masses get a contribution that resembles that of winding states in string theory. This contribution to the square of the mass is negative and for small values of the noncommuting parameter it could dominate over the tree level term.

We also examined a U(1) gauge theory compactified on $T_{\theta}^{2}$. Similar phenomena to those that occur in the scalar case were found.

\section{Acknowledgments}

We would like to thank Hirosi Ooguri for reading an earlier draft of this paper and Edward Witten for useful comments. J.G. would like to thank CERN for hospitality during the final stages of this work. J.G. and T.M. are supported in part by the DOE under grant no. DE-FG03-92-ER 40701. 


\section{References}

[1] S. Minwalla, M.V. Raamsdonk and N. Seiberg, "Noncommutative Perturbative Dynamics", hep-th/9912072.

[2] M.V. Raamsdonk and N. Seiberg, "Comments on Noncommutative Perturbative Dynamics", hep-th/0002186.

[3] M. Hayakawa, "Perturbative analysis on infrared and ultraviolet aspects of noncommutative QED on $R^{4}$," hep-th/9912167.

[4] W. Fischler, E. Gorbatov, A. Kashani-Poor, S. Paban, P. Pouliot and J. Gomis, "Evidence for winding states in noncommutative quantum field theory," hep-th/0002067.

[5] A. Matusis, L. Susskind and N. Toumbas, "The IR/UV connection in the noncommutative gauge theories," hep-th/0002075.

[6] T. Filk, "Divergences in a Field Theory on Quantum Space", Phys. Lett. B376 53 (1996).

[7] J.C. Varilly and J.M. Gracia-Bondia, "On the ultraviolet behaviour of quantum fields over noncommutative manifolds", Int. J. Mod. Phys. A14 (1999) 1305, hepth/9804001.

[8] M. Chaichian, A. Demichev and P. Presnajder, "Quantum Field Theory on Noncommutative Space-times and the Persistence of Ultraviolet Divergences", hepth/9812180; "Quantum Field Theory on the Noncommutative Plane with E(q)(2) Symmetry", hep-th/9904132.

[9] C.P. Martin, D. Sanchez-Ruiz, "The One-loop UV Divergent Structure of U(1) YangMills Theory on Noncommutative $R^{4}$ ", Phys. Rev. Lett. 83 (1999) 476-479, hepth/9903077

[10] M. Sheikh-Jabbari, "One Loop Renormalizability of Supersymmetric Yang-Mills Theories on Noncommutative Torus", JHEP 06 (1999) 015, hep-th/9903107; "Noncommutative Super Yang-Mills Theories with 8 Supercharges and Brane Configurations", hep-th/0001089.

[11] I.Ya. Areféva, D.M. Belov and A.S. Koshelev, "Two-Loop Diagrams in Noncommutative $\phi_{4}^{4}$ theory", hep-th/9912075; "A Note on UV/IR for Noncommutative Complex Scalar Field", hep-th/0001215; "UV/IR Mixing for Noncommutative Complex Scalar Field Theory, II (Interaction with Gauge Fields)", hep-th/0003176.

[12] H. Grosse, T. Krajewski and R. Wulkenhaar, "Perturbative quantum gauge fields on the noncommutative torus", hep-th/9903187; "Renormalization of noncommutative Yang-Mills theories: A simple example", hep-th/0001182.

[13] S. Cho, R. Hinterding, J. Madore and H. Steinacker, "Finite Field Theory on Noncommutative Geometries", hep-th/9903239.

[14] E. Hawkins, "Noncommutative Regularization for the Practical Man", hep-th/9908052. 
[15] I. Chepelev and R. Roiban, "Renormalization of Quantum Field Theories on Noncommutative $R^{d}$, I. Scalars," hep-th/9911098.

[16] H.O. Girotti, M. Gomes, V.O. Rivelles and A.J. da Silva, "A Consistent Noncommutative Field Theory: the Wess-Zumino Model", hep-th/0005272.

[17] S.S. Gubser and S.L. Sondhi, "Phase structure of non-commutative scalar field theories", hep-th/0006119.

[18] J. Gomis and T. Mehen, "Space-Time Noncommutative Field Theories and Unitarity", hep-th/0005129.

[19] A. Connes, M.R. Douglas and A. Schwarz, "Noncommutative Geometry and Matrix Theory: Compactification on Tori", JHEP 9802(1998) 003, hep-th/9711162.

[20] M.R. Douglas and C. Hull, "D-branes and the Noncommutative Torus", JHEP 9802 (1998) 008, hep-th/9711165.

[21] N. Seiberg and E. Witten, "String Theory and Noncommutative Geometry", JHEP 9909 (1999) 032, hep-th/9908142.

[22] Y.-K. E. Cheung and M. Krogh, "Noncommutative Geometry From 0-Branes In A Background B Field", Nucl. Phys. B528 (1998) 185, hep-th/98030031.

[23] C.-S. Chu and P.-M. Ho, "Noncommutative Open String And D-brane", Nucl. Phys. B550 (1999) 151, hep-th/9812219; "Constrained Quantization of open string in background B field and noncommutative D-brane", hep-th/9906192.

[24] V. Schomerus, "D-Branes And Deformation Quantization", JHEP 9906:030 (1999), hep-th/9903205.

[25] F. Ardalan, H. Arfaei and M.M. Sheikh-Jabbari, "Mixed Branes and M(atrix) Theory on Noncommutative Torus", hep-th/9803067; "Noncommutative Geometry From Strings and Branes", JHEP bf 9902 (1999), hep-th/9810072; "Dirac Quantization of Open Strings and Noncommutativity in Branes", hep-th/9906161.

[26] N. Arkani-Hamed, S. Dimopoulos and G. Dvali, "The Hierarchy Problem and New Dimensions at a Millimeter", hep-ph/98033115, Phys. Lett. B429 (1998) 263; I. Antoniadis, N. Arkani-Hamed, S. Dimopoulos and G. Dvali, "New Dimensions at a Millimeter to a Fermi and Superstrings at a TeV", Phys. Lett. B436 (1998) 257; I. Antoniadis, "A Possible New Dimension at a Few TeV", Phys. Lett. B246 (1990) 377; N. Arkani-Hamed, S. Dimopoulos and J. March-Russell, "Stabilization of Submillimeter Dimension: The New Guise of the Hierarchy Problem", hep-th/9809124; L. Randall and R. Sundrum, "A Large Mass Hierarchy from a Small Extra Dimension", hep-th/99050221, Phys. Rev. Lett. 83 (1999) 3370.

[27] N. Arkani-Hamed, S. Dimopoulos, N. Kaloper and R. Sundrum, "A Small Cosmological Constant from a Large Extra Dimension", hep-th/00031197, Phys. Lett. B480 (2000) 193; S. Kachru, M. Schulz and E. Silverstein, "Self Tuning Flat Domain Walls in 5-D Gravity and String Theory", hep-th/0001206.

[28] J.Gomis, M. Kleban, T. Mehen, M. Rangamani and S. Shenker, "Noncommutative Gauge Dynamics from the String World Sheet", hep-th/0003215. 